\title{
A direct contact between astrocyte and vitreous body is possible in the rabbit eye due to discontinuities in the basement membrane of the retinal inner limiting membrane
}

A. Haddad ${ }^{1}$, J.J. Salazar ${ }^{2}$, E.M. Laicine ${ }^{1}$, A.I. Ramírez ${ }^{2}$, J.M. Ramírez ${ }^{2}$ and A. Triviño ${ }^{2}$
Correspondence

A. Haddad

Departamento de Biologia Celular

e Molecular, FMRP, USP

Av. Bandeirantes, 3900

14049-900 Ribeirão Preto, SP

Brasil

Fax: +55-16-633-1786

E-mail: ahaddad@rbp.fmrp.usp.br

Research supported by FAPESP (A. Haddad and E.M. Laicine), CNPq (A. Haddad), and PROINTERUSP (A. Haddad and E.M. Laicine).

Received May 24, 2002 Accepted November 12, 2002 ...................

\begin{abstract}
Different from most mammalian species, the optic nerve of the rabbit eye is initially formed inside the retina where myelination of the axons of the ganglion cells starts and vascularization occurs. Astrocytes are confined to these regions. The aforementioned nerve fibers known as medullated nerve fibers form two bundles that may be identified with the naked eye. The blood vessels run on the inner surface of these nerve fiber bundles (epivascularization) and, accordingly, the accompanying astrocytes lie mostly facing the vitreous body from which they are separated only by the inner limiting membrane of the retina. The arrangement of the astrocytes around blood vessels leads to the formation of structures known as glial tufts. Fragments $(\mathrm{N}=3)$ or whole pieces $(\mathrm{N}=3)$ of the medullated nerve fiber region of threemonth-old male rabbits (Orictolagus cuniculus) were fixed in glutaraldehyde followed by osmium tetroxide, and their thin sections were examined with a transmission electron microscope. Randomly located discontinuities (up to a few micrometers long) of the basement membrane of the inner limiting membrane of the retina were observed in the glial tufts. As a consequence, a direct contact between the astrocyte plasma membrane and vitreous elements was demonstrated, making possible functional interactions such as macromolecular exchanges between this glial cell type and the components of the vitreous body.
\end{abstract}

The axons of the ganglion cells, before becoming the nerve fibers of the optic nerve, are enveloped by a myelin sheath inside the rabbit eye where they are grouped into two bundles of nerve fibers, the medullated nerve fibers, clearly visualized with the naked eye. This is the only region where a partial vascu-
Key words

- Astrocyte

- Basement membrane

- Inner limiting membrane

- Vitreous body

- Rabbit retina

- Plasma membrane larization occurs whereas the remainder of the rabbit retina is avascular. This is also the only retinal region where astrocytes occur (1-5).

It has been known for years that some types of astrocytes are located around or between the vitreous blood vessels that run 
on the inner surface of the medullated nerve fibers. These anatomical structures are referred to as glial tufts and have been described both at the light and at the electron microscope level $(6,7)$. Recently, they were also studied by our group using immunostaining for glial fibrillary acidic protein (GFAP) in association with transmission and scanning electron microscopy (1). Thus, we observed that whole astrocytes lie apparently outside the retinal tissue proper, being located between a blood vessel and the inner limiting membrane. This means that the surface of the astrocyte is separated from the vitreous body only by a very thin basement membrane of the inner limiting membrane of the rabbit retina.

That investigation also provided indications for the occurrence of discontinuities of the inner limiting membrane which would make possible a direct contact between the vitreous body and the astrocyte plasma membrane. Since the retinas used for these studies were fixed only in formaldehyde and subsequently handled for several days for the immunohistochemical technique, the lack of basement membrane at some spots of the retina could be the result of an artifact. Therefore, we decided to re-examine the subject by processing the rabbit eyes according to the protocol described below. The scope of our investigation, however, was to examine this novel morphological finding and not to describe again the cellular composition of the glial tuft.

Three-month-old male albino rabbits (three for each series of experiments) were anesthetized with sodium thiopental injected into the marginal vein of the ear and killed by intracardiac perfusion of a fixative consisting of $2 \%$ glutaraldehyde (electron microscopy grade) plus $2 \%$ formaldehyde (freshly prepared from paraformaldehyde) in $0.1 \mathrm{M}$ Sorensen's phosphate buffer, $\mathrm{pH}$ 7.2, at room temperature $\left(26-27^{\circ} \mathrm{C}\right)$, for $15 \mathrm{~min}$. After perfusion, the eyes were enucleated and cut behind the limbus with a sharp razor blade in order to facilitate penetration of the fixative. The eyes were immersed in the same fixative and kept at $4^{\circ} \mathrm{C}$ for $5 \mathrm{~h}$. After washing in 0.1 $\mathrm{M}$ phosphate buffer, the wall of the posterior segment of the eye was cut into small pieces comprising all regions of the retina. These fragments were refixed in $1 \%$ osmium tetroxide in $0.1 \mathrm{M}$ phosphate buffer for $2 \mathrm{~h}$ at $4^{\circ} \mathrm{C}$. Then, the tissues were dehydrated in graded ethanol, cleared in propylene oxide and embedded in LX112 resin (Ladd Research Ind., Burlington, VT, USA).

In another series of experiments, the medullated nerve fiber region of the rabbit retina was carefully dissected with a minimum of manipulation on the attached vitreous body and processed in toto for resin embedding as above. After completion of polymerization, the blocks containing the medullated nerve fibers were cut perpendicularly with a fine saw and the slices reembedded in the same resin in order to obtain cross-sections of the above retinal region. The semithin sections were stained with toluidine blue and, after selection, the blocks were further trimmed for ultramicrotomy. The thin sections were contrasted with uranyl acetate and lead citrate, and examined with a Philips model 208 transmission electron microscope.

Due to their morphological features and peculiar location (reviewed in 1), the astrocytes were promptly identified on regular electron micrographs of rabbit retinas. They were found only where an epivascularization of the retina occurred, i.e., in the medullated nerve fiber region that indicates the origin of the optic nerve in rabbits. This is also the retinal region where glial tufts were observed, and it is known that astrocytes are the main glial cell type found in these tufts. The astrocytes were also located in the retinal core where glial tufts did not occur. Besides the bundles of cytoplasmic intermediate filaments (known to contain GFAP), it is noteworthy that rabbit astrocytes contained many lysosomes and complex dense bodies indi- 
cating intracellular digestive activities (Figure 1). Glycogen granules were sparse and scarce not only in the peripheral processes (Figure 1, inset) but also in other regions of all astrocytes including those more internally located in the retina of the rabbits. Together with GFAP-containing intermediate filaments, glycogen granules are a morphological feature of astrocytes. Several types of cell junctions, notably gap junctions, were conspicuous in the glial tufts, a clear indication that they are made up by several astrocytes (Figures 1 and 2). As reported previously, observations on semithin resin sections and on specimens prepared for transmission and scanning electron microscopy indicated quite clearly that some of the rabbit astrocytes are apparently located outside the retina. This was based on observations of whole astrocytes abutting the vitreous body, that is, they were located between the vitreous body and the wall of small blood vessels running longitudinally on the medullated nerve fibers (1-5).

Examination of 10 to 20 electron micrographs per eye revealed that the basement membrane of the inner limiting layer was continuous, without any gap, all over the rabbit retina except in some regions where astrocytes were found projecting toward the vitreous body, e.g., the glial tufts. The discontinuity of the basement membrane was observed not only on the tips of astrocyte microvilli but also in those regions where a direct contact of the astrocyte surface with the vitreous elements was not so conspicuous (Figures 1 and 2). This was detected in all six rabbits used for the present report. Glial cells of the oligodendrocyte lineage (6) were not obvious on regular electron micrographs.

In view of the good quality of the tissue fixation and the reproducible findings in all six rabbits, the discontinuity of the basement membrane, as described above, can hardly be explained by the occurrence of artifacts during the preparation of the retinas for elec-

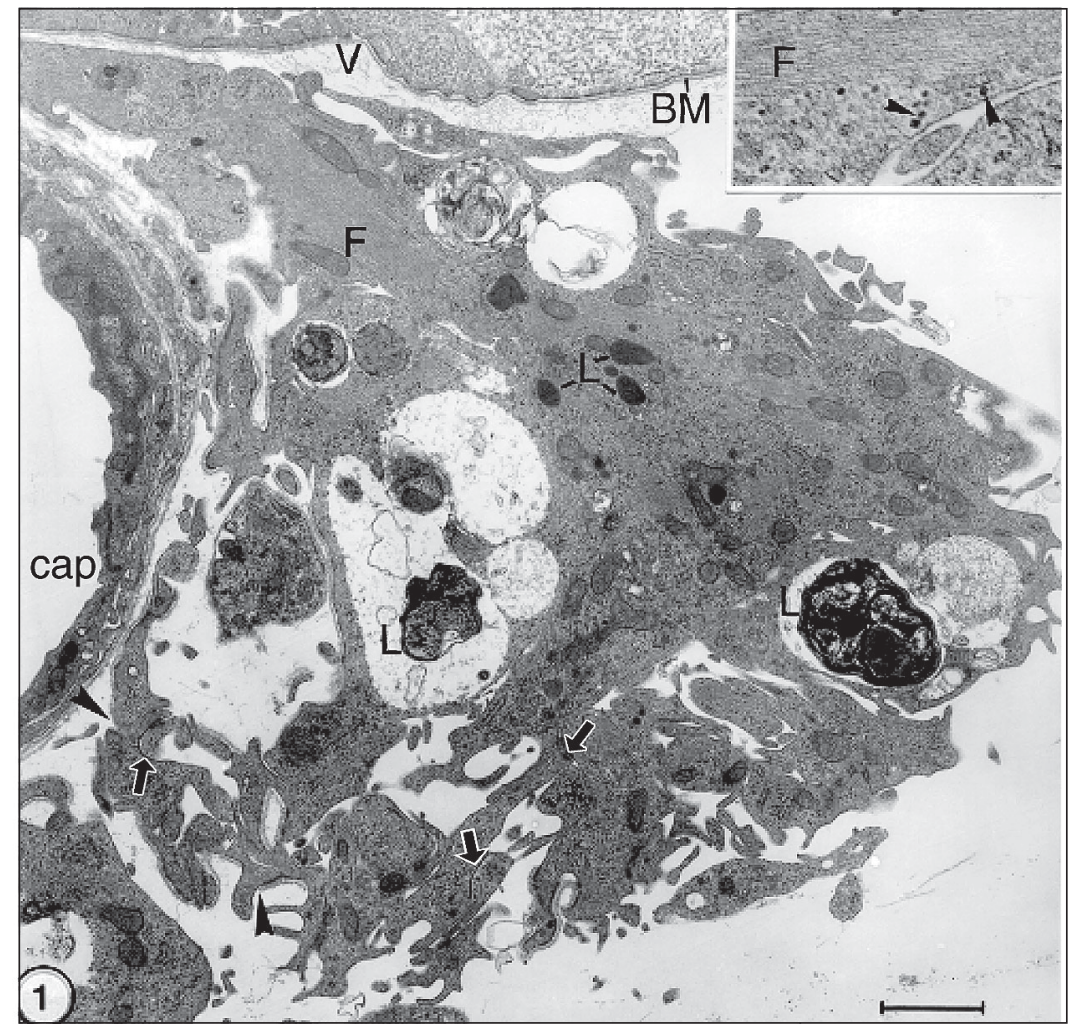

Figure 1. Electron micrograph of the rabbit retina at the site of a glial tuft. Contrast with uranyl acetate and lead citrate. Bar $=1 \mu \mathrm{m}$. The regular retina is seen in the upper portion with a continuous inner limiting membrane with its basement membrane (BM) separating the retina from the vitreous body (V). Most of the figure is occupied by a glial tuft. Part of a capillary (cap) is observed on the left. Portions of several astrocytes may be identified by cytoplasmic bundles of intermediate filaments (F). Cell junctions are indicated by arrows. Note lysosomes (L) in the astrocyte cytoplasm. Even at this relatively low magnification, interruptions of the basement membrane around the astrocytes may be observed (arrowheads). The inset at the upper right corner shows a peripheral process with glycogen granules (arrowheads) close to intermediate filaments (F). 40,000X.

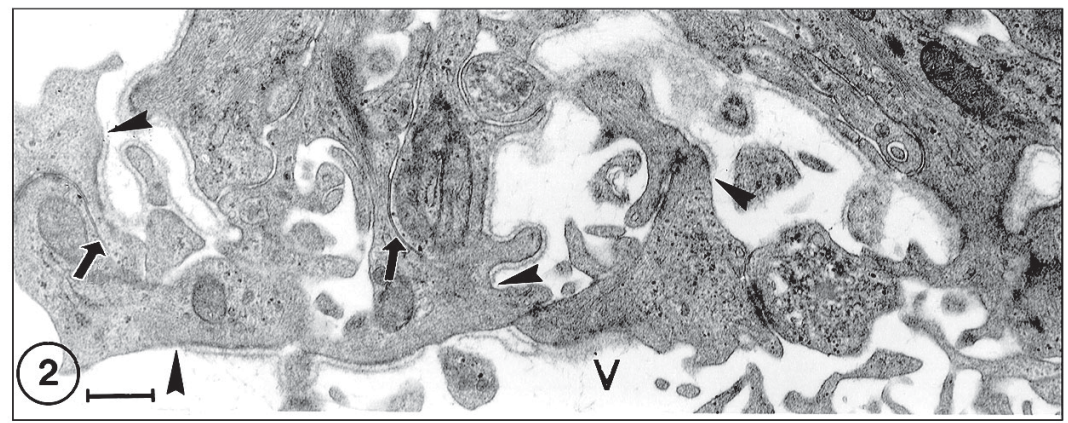

Figure 2. High power view of a glial tuft abutting the vitreous body (V). Bar $=0.5 \mu \mathrm{m}$. Note gap junctions (arrows) and discontinuities of the basement membrane around the astrocytes (arrowheads). 
tron microscopy examination. The discontinuity was observed at random sites and therefore was not due to mishandling, such as inadvertent pulling of the vitreous body during dissection of the eye. Such discontinuities were detected both when the retinas were chopped into small fragments and when the medullated nerve fiber regions were processed in toto. Therefore, a direct contact between the vitreous body and the plasma membrane of the astrocytes possibly occurs.

The only known cell type found inside the vitreous matrix is the hyalocyte (8) and its role in the vitreous physiology is still debatable. Now, we are describing a situation in which interactions between the plasma membrane of another cell type and vitreous macromolecular compounds may occur.

The functional meaning of this unusual location of astrocytes cannot be unraveled by morphological methods only. Therefore, we are performing additional experiments aimed at clarifying the interactions between vitreous body and astrocytes. In one of them, slices of retina with or without astrocytes were assayed for transferrin synthesis and secretion. While synthesis occurred in all slices, transferrin secretion was observed only in the astrocyte-containing retina, an indication that, together with oligodendrocytes and the ciliary body $(9,10)$, the astrocytes may be a source of vitreous transferrin. In fact, experiments carried out with cells in culture have demonstrated transferrin secre- tion by both astrocytes and oligodendrocytes $(11,12)$. These investigations also revealed that these cell types contain transferrin messenger RNA. Experiments are also being designed to verify if the astrocytes are involved in the digestion and elimination of vitreous components in view of the ubiquity of digestive vacuoles in their cytoplasm. However, these experiments have been difficult to carry out in view of the toxicity of the usual tracers (particularly ferritin) for the vitreous body. Another point to be considered is the unusual pattern of vascularization of the rabbit retina which might be a consequence of the also unusual pattern of formation of the optic nerve in this animal species. In this case, the astrocytes around the blood vessels (1) would be required for the formation of the blood-brain barrier (13) that is essential for the proper functioning of the retina.

Finally, this peculiar arrangement of astrocytes in the rabbit retina could indicate that the astrocytes are actually located outside the central nervous system.

\section{Acknowledgments}

The authors express their gratitude to José A. Maulin, Maria D.S. Ferreira and Domingos S. Souza Filho, Departamento de Biologia Celular e Molecular e Bioagentes Patogênicos, for technical assistance.

\section{References}

1. Haddad A, Ramírez Al, Laicine EM, Salazar JJ, Triviño A \& Ramírez JM (2001). Immunohistochemistry in association with scanning electron microscopy for the morphological characterization and location of astrocytes of the rabbit retina. Journal of Neuroscience Methods, 106: 131-137.

2. Hyvärinen $L$ (1967). Vascular structures of the rabbit retina. Acta Ophthalmologica, 45: 852-861.

3. Robinson SR \& Dreher Z (1989). Evidence for three morphological classes of astrocyte in the adult rabbit retina: functional and developmental implications. Neuroscience Letters, 106: 261-268.

4. Schnitzer J \& Karschin A (1986). The shape and distribution of astrocytes in the retina of the adult rabbit. Cell and Tissue Research,
246: 91-102.

5. Triviño A, Ramírez JM, Ramírez Al, Salazar JJ \& García-Sánchez J (1992). Retinal perivascular astroglia: an immunoperoxidase study. Vision Research, 32: 1601-1607.

6. Morcos $Y$, Shorey CD \& Chan-Ling $T$ (1999). Contribution of $\mathrm{O}^{+}$ oligodendrocyte precursors and astrocytes to the glial ensheathment of vessels in the rabbit myelinated streak. Glia, 27: 1-14.

7. Tripathi B \& Ashton N (1971). Vaso-glial connections in the rabbit retina. British Journal of Ophthalmology, 55: 1-11.

8. Haddad A \& André JC (1998). Hyalocyte-like cells are more numerous in the posterior chamber than they are in the vitreous of the rabbit eye. Experimental Eye Research, 66: 709-718. 
9. Laicine EM \& Haddad A (1994). Transferrin, one of the major vitreous proteins, is produced within the eye. Experimental Eye Research, 59: 441-446.

10. Rodrigues MLP, Bertazolli-Filho R, Laicine EM \& Haddad A (1998). Transferrin production by the ciliary body of rabbits: a biochemical and immunocytochemical study. Current Eye Research, 17: 694699.

11. Espinosa de los Monteros A, Kumar S, Scully S, Cole R \& de Vellis J
(1990). Transferrin gene expression and secretion by rat brain cells in vitro. Journal of Neuroscience Research, 25: 576-580.

12. Zahs KR, Bigornia V \& Deschepper CF (1993). Characterization of "plasma proteins" secreted by cultured rat macroglial cells. Glia, 7: 121-133.

13. Janzer RC \& Raff MC (1987). Astrocytes induce blood-brain barrier properties in endothelial cells. Nature, 325: 253-257. 